\title{
Estudo da mastigação e da deglutição em crianças e adolescentes com Sequência de Möbius
}

\author{
Study of chewing and swallowing in children and teenagers with \\ Möbius syndrome
}

\author{
Mariana Saconato ${ }^{1}$ Zelita Caldeira Ferreira Guedes ${ }^{2}$
}

\begin{abstract}
RESUMO
Objetivos: Descrever as alterações morfofuncionais nas funções de mastigação e deglutição, reconhecer as consistências alimentares mais bem aceitas e avaliar a presença de manobras compensatórias durante a deglutição em crianças com Sequência de Möbius. Métodos: A casuística consistiu de oito crianças, sendo três do sexo feminino e cinco do sexo masculino, na faixa etária de cinco a 15 anos, diagnosticadas com Sequência de Möbius. As crianças foram avaliadas em situação de alimentação, nas quais foram oferecidos alimentos de consistência líquida, pastosa e sólida. Os dados coletados foram registrados em protocolo de avaliação adaptado por Guedes, Shintani e Cabello (2003). Resultados: Os resultados obtidos demonstraram significância entre a condição de mobilidade de língua e o tipo de mastigação. As variáveis velocidade de mastigação e contração do masseter, também se mostraram dependentes ( $\mathrm{p}=0,018)$, uma vez que não houve alteração da velocidade da mastigação quando a contração do masseter estava presente. $O$ pão de queijo mostrou-se como a melhor consistência sólida, pois produziu um bolo mais coeso e menos espalhado na cavidade oral. Conclusões: As alterações morfofuncionais presentes nos pacientes com Sequência de Möbius contribuíram para a alteração da dinâmica da mastigação e deglutição. Toda a amostra fez uso de manobras compensatórias e, mesmo com a ausência dos sinais clínicos sugestivos de penetração/aspiração, estes pacientes devem ser submetidos à terapia fonoaudiológica, a fim de adequar os órgãos fonoarticulatórios na tentativa de proporcionar uma refeição mais prazerosa.
\end{abstract}

Descritores: Síndrome de Möbius; Sistema estomatognático; Deglutição; Mastigação; Transtornos de deglutição

\section{INTRODUÇÃO}

A mastigação é uma das funções mais importantes do sistema estomatognático. Para que seja efetiva, é necessário que haja contração coordenada de vários grupos musculares, sendo os mastigatórios os mais destacados, embora também sejam fundamentais os músculos da boca e os faciais, em especial, o bucinador e orbicular dos lábios. A diminuição do tônus muscular dos órgãos fonoarticulatórios, a restrição dos movimentos mandibulares e a alteração das praxias orofaciais tornam os movimentos mastigatórios incompetentes ${ }^{(1-3)}$.

Os músculos envolvidos na mastigação são chamados de músculos mastigatórios. Estes músculos podem ser divididos em levantadores ou depressores da mandíbula, dependendo

Trabalho realizado no Departamento de Fonoaudiologia da Universidade Federal de São Paulo - UNIFESP - São Paulo (SP), Brasil.

(1) Fonoaudióloga clínica - São Paulo (SP), Brasil.

(2) Professora Associada da Disciplina de Distúrbios da Comunicação Humana do Departamento de Fonoaudiologia da Universidade Federal de São Paulo - UNIFESP - São Paulo (SP), Brasil.

Endereço para correspondência:

Mariana Saconato. R. Nelson Faria Mendes, 23, Jd. Três Marias, São Paulo - SP, CEP 03677-130. E-mail: marisac35@yahoo.com.br

Recebido em: 18/4/2008; Aceito em: 21/1/2009 da sua ação durante a mastigação. Os músculos levantadores da mandíbula são: temporal, masséter e pterigóideo medial e os depressores: pterigóideo lateral e toda a musculatura supra-hióidea (principalmente o ventre anterior do digástrico, o gênio-hióideo e o milo-hióideo). Todos estes músculos são inervados pelo ramo motor do $5^{\circ}$ par craniano, sendo o processo da mastigação controlado por núcleos do tronco cerebral $^{(4)}$.

A deglutição, por sua vez, tem como função fundamental a propulsão do alimento da boca para o estômago. É um processo contínuo no qual todos os músculos relacionados com a cavidade oral entram em função ${ }^{(4-5)}$.

A deglutição pode ser dividida em quatro fases: (1) fase oral preparatória; (2) fase oral (ou de transporte); (3) fase faríngea e (4) fase esofágica. As duas primeiras fases (1) e (2) são voluntárias, enquanto que as fases faríngea e esofágica, são involuntárias. A fase oral preparatória da deglutição é uma fase mecânica e envolve a transformação do alimento do estágio sólido para uma fragmentação ${ }^{(6-7)}$.

A fase oral prepara o alimento para a deglutição, envolvendo para isso a coordenação dos seguintes movimentos: fechamento dos lábios para segurar o alimento na porção anterior da boca, tensão da musculatura labial e bucal, para 
que ocorra o fechamento dos sulcos lateral e anterior, movimento de rotação lateral da mandíbula para a execução da mastigação e, finalmente, o movimento de rotação lateral da língua para posicionar o alimento entre as arcadas durante a mastigação ${ }^{(7-8)}$.

A fase oral tem início com a propulsão posterior da língua, cujo perímetro se mantém em contato com os rebordos alveolares. Em seguida, a ponta da língua se eleva até o contato com a papila retroincisal, seu corpo desliza contra o palato duro, e a base da língua se deprime. A cavidade oral é fechada anteriormente pela aproximação dos lábios e elevação da mandíbula. Nesse momento, tem-se a contração do milohióideo, que permite uma elevação maciça da língua e, então, o bolo é direcionado para a orofaringe. Nos indivíduos normais, no momento em que a língua entra em contato com o palato duro até o movimento para cima do hióide, tem-se o início da fase faríngea e estas ações passam-se em um segundo ${ }^{(7-9)}$.

A fase faríngea da deglutição é involuntária, com contração dos músculos supra - hióideos, faríngeos, laríngeos e linguais, resultando em alongamento da faringe e interrupção da respiração. Tem início na elevação do palato mole, em direção à parede posterior da rinofaringe, constituindo funcionalmente uma região esfincteriana, que impede a dissipação da pressão do transporte do bolo para a rinofaringe e as fossas nasais ${ }^{(9-10)}$. A elevação e a anteriorização laríngea são muito importantes na proteção da via aérea, pois deslocam a laringe para uma posição mais próxima da base da língua e aumentam o espaço na faringe para a progressão do bolo alimentar, além de desviálo para os recessos piriformes. A contração faríngea facilita $\mathrm{o}$ movimento do bolo para o esôfago. Entretanto, outros fatores, como a propulsão lingual, a gravidade e a elevação laríngea, transportam o bolo na direção craniocaudal ${ }^{(10-11)}$.

A fase esofágica tem início quando o bolo alimentar passa para a transição faringo-esofágica e tem duração de três a nove segundos. Nesta fase, há um relaxamento da musculatura supra-hióidea e a laringe retorna à posição de repouso. $\mathrm{O}$ véu do palato, a língua e a mandíbula também voltam para a posição habitual, reiniciando a respiração. O transporte esofagiano envolve peristalse do corpo de esôfago e termina com o relaxamento do esfíncter inferior esofágico e a passagem do bolo para o estômago ${ }^{(12)}$.

A Sequência de Möbius é uma condição pouco comum caracterizada por uma paralisia total ou parcial dos VI e VII pares cranianos ${ }^{(13-14)}$.

Não se sabe ainda qual é a origem da doença, mas em nosso meio, cerca de $90 \%$ dos casos é consequência de um fator ambiental que ocorre no útero, decorrente do consumo de misoprostol ${ }^{(15-16)}$.

O misoprostol pode favorecer uma contração uterina que leva a um decréscimo do fluxo sanguíneo, associada a uma flexão do embrião na região dos pares cranianos, facilitando a ocorrência de uma lesão por hemorragia na região do VI e VII pares ${ }^{(14)}$.

As principais estruturas acometidas e as alterações mais comuns do portador da Sequência de Möbius são: crânio com fronte achatada e estreita, face com paralisia bilateral, sem rugas de expressão, olhos com estrabismo convergente, ptose palpebral, pregas epicânticas e hipertelorismo. Nas orelhas, podem ocorrer alterações variadas no pavilhão auricular. Quanto aos aspectos otológicos, há ausência de reflexos acústicos (devido à paralisia do nervo facial). Os lábios geralmente são pequenos, com queda das comissuras e encontram-se permanentemente abertos e sem mobilidade (inabilidade para sorrir, assobiar e elevar o lábio superior). A sialorréia pode estar presente, além da microglossia e anquiloglossia. Nos dentes, pode haver hipoplasia dentária e oclusão tipo Classe II de Angle. Geralmente, o palato duro é profundo, podendo, em alguns casos, ocorrer fissura palatina ${ }^{(17-19)}$.

No Sistema Nervoso Central, pode haver alterações neurológicas, mas em $90 \%$ dos casos a inteligência está pre$\operatorname{servada}^{(20)}$.

As alterações da mímica, com certeza, vão interferir no processo de comunicação. A dificuldade da mobilidade de língua e palato mole ocasiona dificuldades na produção de fonemas e tais dificuldades favorecem o aparecimento de mecanismos compensatórios, semelhantes aos portadores de fenda palatina, como: golpe de glote, fricativas faríngeas e outros ${ }^{(21)}$.

Considerando as alterações morfofuncionais no sistema estomatognático, julga-se necessário estudar as funções de mastigação e deglutição destes pacientes. Dessa forma, o presente trabalho teve como objetivos: descrever as alterações morfofuncionais no sistema estomatognático, primordialmente das funções de mastigação e deglutição; avaliar as consistências alimentares de melhor aceitação e verificar a presença de manobras compensatórias durante a deglutição.

\section{MÉTODOS}

O presente estudo foi aprovado pela Comissão de Ética em Pesquisa da Universidade Federal de São Paulo - UNIFESP, protocolado sob o número 0532/06.

Para que os pacientes pudessem participar desta pesquisa seus responsáveis leram e assinaram o Termo de Consentimento Livre e Esclarecido juntamente com a autora.

A casuística consistiu de oito crianças na faixa etária de quatro a 15 anos, sendo três do sexo feminino e cinco do sexo masculino, com Sequência de Möbius diagnosticada por neurologistas e/ou geneticistas.

Estas crianças pertencem a um grupo de pacientes com Möbius que é atendido e tratado no Ambulatório do Departamento de Fonoaudiologia da UNIFESP.

As crianças foram avaliadas em situação de alimentação no período de Novembro de 2006 à Abril de 2007 por uma única avaliadora, com supervisão de docente com mais de 20 anos de experiência. Foram oferecidos alimentos de consistência líquida, pastosa e sólida, com diferentes texturas, sabores e temperaturas.

Observou-se os processos de mastigação e deglutição dos alimentos. Para isso, foram extraídos apenas alguns itens presentes no protocolo utilizado no ambulatório de paralisia facial da Universidade Federal de São Paulo ${ }^{(1)}$.

\section{Etapa I - Avaliação dos órgãos fonoarticulatórios}

Esta etapa consistiu na avaliação dos seguintes órgãos fonoarticulatórios: lábios; língua e mandíbula. 
Todos foram submetidos a uma avaliação que constou das seguintes etapas:

\section{Lábios}

A avaliação dos lábios tratou de verificar a mobilidade e a tonicidade labial.

Mobilidade labial

Neste item, o paciente deveria imitar os gestos que a pesquisadora realizava com os lábios. Foi solicitado:

- Projetar os lábios unidos (como um beijo) e em seguida estirá-los (como em um sorriso), mantendo os dentes ocluídos;

- Movimentar os lábios unidos em direção à comissura labial do lado direito e depois do lado esquerdo.

Considerou-se:

a- Adequada, quando os participantes realizaram os movimentos citados acima adequadamente.

b- Diminuída, quando estes não foram capazes de realizar corretamente um ou mais movimentos.

Resistência labial

- Foi solicitado aos pacientes manter entre os lábios um depressor de língua de madeira, exercendo sobre ele a maior pressão possível (para mantê-lo nessa posição, durante cinco segundos);

- Com o depressor de língua de madeira, a avaliadora exerceu uma pressão sobre os lábios dos pacientes que se encontravam projetados e unidos (como um beijo) e solicitou para que fizessem uma força contrária ao do pressionamento. Considerou-se:

a- Adequada, quando os pacientes realizaram os movimentos anteriormente citados, adequadamente.

b- Diminuída, quando estes não foram capazes de realizar corretamente um ou mais movimentos.

\section{Língua}

A avaliação da língua incluiu os itens referentes à mobilidade e resistência.

Mobilidade lingual

A pesquisadora fornecia as instruções verbais dos movimentos que os pacientes deveriam realizar com a língua. Além dessas instruções, a pesquisadora realizava os movimentos que deveriam ser imitados.

Os pacientes deveriam imitar os seguintes movimentos:

- Tocar com a ponta da língua as comissuras labiais: direita e esquerda;

- Tocar com a ponta da língua o lábio superior e inferior;

- Estalar a ponta da língua contra o palato duro anterior. Considerou-se:

a- Adequada, quando os pacientes realizaram os movimentos anteriormente citados, adequadamente.

b- Diminuída, quando estes não foram capazes de realizar corretamente um ou mais movimentos.

\section{Resistência lingual}

Para a observação deste item, o paciente estirava a língua fora da cavidade oral. A pesquisadora pressionava a ponta da língua com um depressor de madeira (no sentido vertical) e solicitava ao paciente que realizasse força no sentido contrário.
Considerou-se:

a- Adequada, quando os pacientes apresentavam resistência à pressão imposta pela avaliadora (com depressor lingual);

b- Diminuída, à ausência ou diminuição da resistência à pressão imposta pela avaliadora (com depressor lingual).

\section{Mandíbula}

A avaliação da mandíbula tratou de verificar a mobilidade e tonicidade dos músculos masseter e temporal.

Mobilidade da mandíbula

Neste item, o paciente deveria imitar os gestos que a pesquisadora realizava com a mandíbula. Foi solicitado:

- Abrir e fechar a boca;

- Abrir e fechar a boca contra resistência;

- Levar a mandíbula para a direita;

- Levar a mandíbula para a esquerda;

- Levar a mandíbula para frente;

- Levar a mandíbula para trás;

- Realizar movimentos circulares da mandíbula.

Considerou-se:

a- Adequada, quando os pacientes realizaram os movimentos anteriormente citados, adequadamente.

b- Diminuída, quando estes não foram capazes de realizar corretamente um ou mais movimentos.

\section{Tonicidade dos músculos masseter e temporal}

Para avaliar os músculos masseter e temporal, a avaliadora palpou a região destes músculos durante a mastigação do pão de queijo.

Considerou-se:

a- Adequada, quando o paciente foi capaz de contrair os músculos masseter e temporal;

b- Inadequada, quando o paciente não foi capaz de contrair os músculos masseter e temporal.

\section{Etapa II - Avaliação das funções orais}

A segunda parte do protocolo consistiu na avaliação das seguintes funções orais: mastigação e deglutição.

Os procedimentos clínicos utilizados nas avaliações de cada função foram os seguintes:

\section{Mastigação}

Para a avaliação da mastigação, foram oferecidas duas porções de 0,05 gramas de cada um dos seguintes alimentos: pão francês, pão de queijo e biscoito waffer. A função mastigatória foi avaliada quanto ao seu tipo, modo e velocidade, por um examinador supervisionado por docente com mais de 20 anos de experiência.

Considerou-se o tipo mastigatório com relação à posição em que o alimento foi triturado nas arcadas dentárias e classificou-se em:

- unilateral: quando a mastigação era realizada somente em um dos lados das arcadas dentárias;

- bilateral: quando a mastigação era realizada dos dois lados das arcadas dentárias;

Para averiguar o lado em que a mastigação se realizava, a pesquisadora e orientadora fizeram uso do conhecimento 
prévio que tinham da amostra, uma vez que $60 \%$ da mesma tiveram preferência unilateral da musculatura da face e da cavidade oral, através das quais realizavam os movimentos compensatórios. A fim de confirmar tal achado, a avaliadora posicionava a mão na zona do músculo masseter e, dessa forma, observava o lado em que o alimento estava sendo triturado.

O modo mastigatório foi classificado, de acordo com a posição dos lábios durante a mastigação, em:

a) com vedação labial;

b) sem vedação labial.

Para averiguar o modo mastigatório em que a mastigação se realizava, a pesquisadora verificou se os pacientes tiveram o vedamento labial durante a mastigação ou se o realizavam com os dedos no momento da deglutição.

A velocidade de mastigação foi classificada em: normal, aumentada ou diminuída. Para a avaliação da velocidade da mastigação foi utilizado um cronômetro, através do qual foi mensurado o tempo de mastigação para a oferta de 0,05 gramas de cada um dos seguintes alimentos: pão francês, pão de queijo e biscoito waffer.

Em seguida, foi obtida uma média do tempo gasto para a mastigação de cada alimento em questão, sendo: 15s, 10s e 7s para o pão francês, pão de queijo e biscoito waffer respectivamente.

A velocidade da mastigação foi classificada em:

a) normal: quando o tempo para completar a mastigação foi igual ao tempo médio encontrado para cada alimento.

b) aumentada: quando o tempo para completar a mastigação foi maior do que o tempo médio encontrado para cada alimento.

c) reduzida: quando o tempo para completar a mastigação foi menor do que o tempo médio encontrado para cada alimento.

\section{Deglutição}

A avaliação da deglutição foi realizada a partir do protocolo de avaliação elaborado por Guedes, Shintani e Cabello ${ }^{(1)}$.

Para a avaliação a partir do protocolo foram utilizados os seguintes alimentos: água em temperatura ambiente, iogurte com consistência pastosa, pão francês, bolacha waffer e pão de queijo.

Durante a deglutição foi observado se os pacientes:

- empurravam o alimento com o dedo a fim de facilitar a ejeção do alimento;

- se havia protrusão de língua com escape anterior do alimento durante a deglutição;

- se havia movimentação de cabeça para trás a fim de facilitar o trânsito oral do alimento;

- $\quad$ se a propulsão do bolo era eficiente, ou seja, se não havia estase em cavidade oral após a deglutição;

- $\quad$ se a formação do bolo era coesa, ou seja, se o bolo não ficava espalhado na cavidade oral antes da deglutição. Considerou-se a deglutição:

a) Eficiente, quando os pacientes não empurravam o alimento com o dedo e não havia protrusão de língua nem movimentação de cabeça para trás, além de propulsão do bolo eficiente e formação do bolo coesa. b) Ineficiente, quando os pacientes empurravam o alimento com o dedo, com protrusão de língua e movimentação de cabeça durante a deglutição, além de propulsão do bolo ineficiente e formação do bolo não coesa.

\section{RESULTADOS}

Para este trabalho foi utilizado o teste Qui-quadrado com um nível de significância de 0,15 , pouco acima do usualmente utilizado (5\%), pois a amostragem do trabalho foi relativamente baixa, mas considerável, tratando-se de portadores da Síndrome de Möbius.

A análise da Figura 1 mostrou que, para o pão francês e para o biscoito waffer, houve relação estatisticamente significante entre mobilidade normal de língua e mastigação bilateral $(\mathrm{p}=0,102)$, e entre mobilidade de língua alterada e mastigação unilateral $(\mathrm{p}=0,102)$ para ambas as consistências.

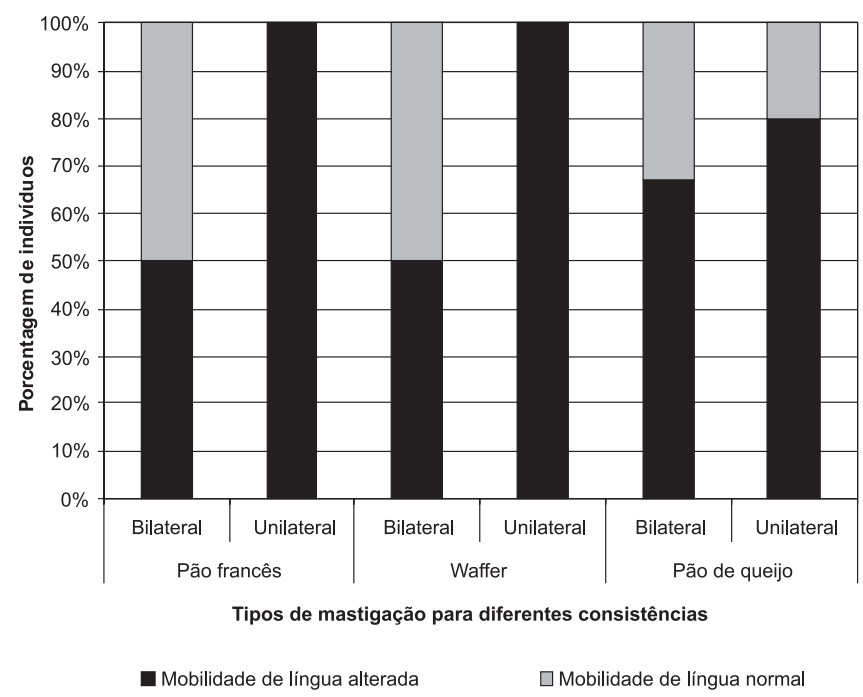

Figura 1. Relação da mobilidade de língua com o tipo de mastigação para os diferentes tipos de consistências alimentares

Nesta mesma figura, pode-se verificar que, quanto à análise da mastigação do pão de queijo, não foi encontrada relação estatisticamente significante entre tipo de mordida (unilateral ou bilateral) e mobilidade de língua (alterada ou normal) $(\mathrm{p}=0,673)$.

Na Figura 2 não se constatou relação estatisticamente significante entre mobilidade de mandíbula e velocidade de mastigação $(p=0,710)$, sendo que $55 \%$ da amostra com velocidade de mastigação diminuída tiveram a mobilidade de mandíbula alterada. Além disso, a análise mostra que dos pacientes que possuíam a mobilidade de mandíbula normal, 100\% tiveram a velocidade de mastigação reduzida.

Na Figura 3 é apresentada a relação da velocidade de mastigação com a contração do masseter para as diferentes consistências alimentares. Os resultados mostraram que as variáveis velocidade de mastigação e contração do masseter são dependentes, com resultados estatisticamente significantes $(\mathrm{p}=0,018)$, uma vez que, nos pacientes em que foi observada a contração deste músculo, a velocidade de mastigação estava normal, independente da consistência alimentar oferecida. En- 


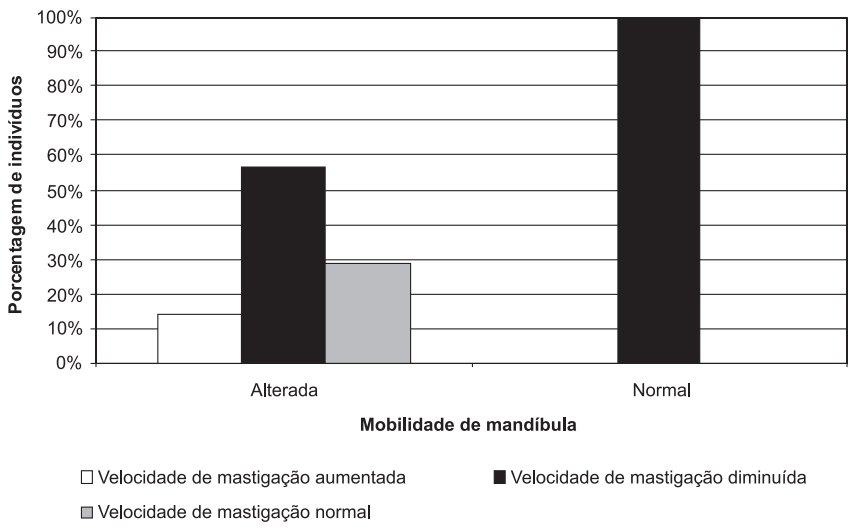

Figura 2. Relação da mobilidade da mandíbula com a velocidade de mastigação

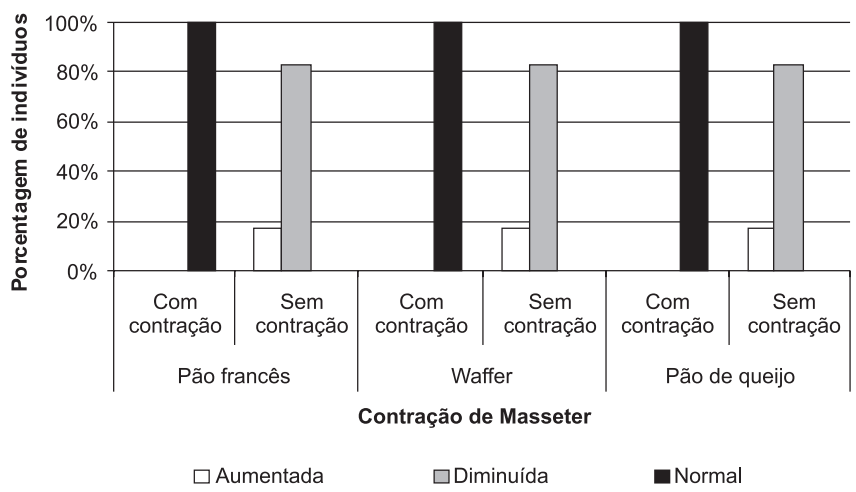

Figura 3. Relação da velocidade de mastigação com a contração do masséter para as diferentes consistências alimentares

tretanto, naqueles pacientes em que a velocidade de mastigação estava diminuída, constatou-se que a contração do músculo masséter estava inadequada.

$\mathrm{Na}$ Figura 4 foi mostrada a relação do tipo de bolo alimentar produzido, de acordo com a consistência alimentar oferecida. Os resultados estatisticamente significantes $(\mathrm{p}=0,018)$ mostraram que os pacientes não se beneficiaram da consistência alimentar produzida pelo biscoito waffer e pelo pão francês, pois $62,5 \%$ dos pacientes produziram bolo espalhado na cavidade oral. Quando ofertado o pão de queijo, $62,5 \%$ dos pacientes produziram um bolo alimentar coeso.

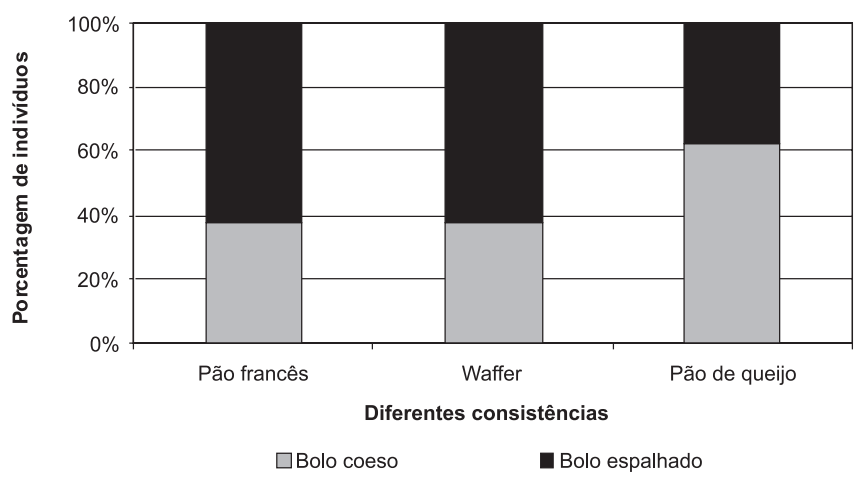

Figura 4. Relação do tipo de bolo alimentar produzido de acordo com a consistência alimentar oferecida
Na Figura 5 as variáveis tonicidade e protrusão da língua durante a mastigação das diferentes consistências alimentares não se mostraram estatisticamente significantes $(\mathrm{p}=0,465)$, uma vez que foi encontrada protrusão de língua em pacientes sem alteração de tônus muscular.

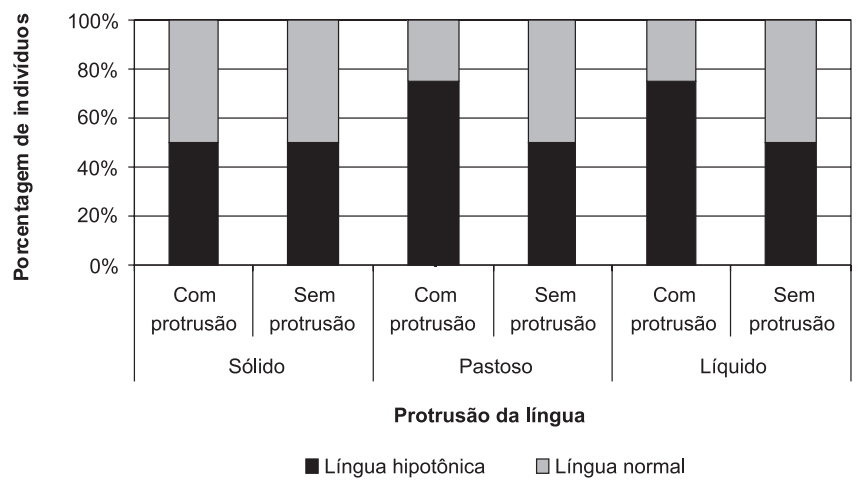

Figura 5. Relação da tonicidade da língua com a protrusão da língua para as diferentes consistências alimentares

A protrusão da língua fez com que houvesse escape do alimento para a região das comissuras labiais. Estes pacientes não conseguiram fazer o vedamento labial; dessa forma, mostraram uma inabilidade em manter a pressão na região intra-oral.

A deglutição mostrou-se ineficiente em 100\% da amostra, visto que todos os pacientes fizeram uso de manobras compensatórias a fim de aumentar o trânsito orofaríngeo, facilitar o início da fase faríngea e eliminar a estase em cavidade oral.

Para alcançar estes objetivos, durante a mastigação e deglutição de pastosos e sólidos, os pacientes empurraram o alimento com o auxílio dos dedos, inclinaram a cabeça para trás e retiraram estase do vestíbulo oral e lateral com os dedos. Durante a deglutição de líquidos, toda a amostra inclinou a cabeça para trás a fim de desencadear a deglutição faríngea e conter a incontinência oral.

Mesmo diante destas manobras compensatórias não foram observados sinais clínicos sugestivos de penetração e aspiração em nenhum paciente.

\section{DISCUSSÃO}

As características morfológicas relacionadas ao sistema estomatognático e o grau da paralisia facial, presentes nos pacientes com Sequência de Möbius comprometem a movimentação e função dos órgãos fonoarticulatórios, uma vez que alterações provocadas por esta doença afetam as seguintes estruturas: lábios (abertos e sem mobilidade), língua (pequena e com diminuição da mobilidade), mandíbula (diminuição da movimentação e da abertura da boca) ${ }^{(21-23)}$.

A mastigação unilateral dos pacientes desta amostra provavelmente deu-se por conta da pouca mobilidade de língua que, por sua vez, ao apresentar paresia, impede o deslocamento do alimento de um lado para o outro na cavidade oral, uma vez que um dos nervos afetados na Sequência de Möbius é o XII par hipoglosso, responsável pela mobilidade deste músculo.

Um estudo realizado com 18 pacientes com diagnóstico de Sequência de Möbius mostrou que o nervo craniano mais 
afetado, após o abducente (VI), é o hipoglosso (XII). Em 11 pacientes da amostra, dois deles tiveram atrofia bilateral e simétrica da língua e nove, envolvimento assimétrico ${ }^{(24)}$.

A baixa mobilidade de mandíbula interferiu na dinâmica da mastigação, porém outros fatores devem ser levados em consideração, quando esta variável é analisada, pois a velocidade de mastigação também foi diminuída, mesmo quando a mobilidade de mandíbula estava normal. Uma possível explicação para este achado deve-se ao comprometimento da mobilidade da língua e hipotonia dos músculos responsáveis pela mastigação.

Sobre os estudos encontrados na literatura, alguns autores sugeriram que uma das estruturas mais comprometidas nos pacientes portadores da Sequência de Möbius é a mandíbula. Segundo estes autores, estes pacientes têm micrognatia, diminuição da movimentação e da massa muscular, além de redução da abertura de boca ${ }^{(25-27)}$.

Quanto à velocidade da mastigação, vimos na Figura 3, que a mesma não teve relação com a mobilidade da língua e da mandíbula; entretanto, está diretamente relacionada à tonicidade e capacidade de contração do masséter.

Estudos mostraram que o músculo masseter, quando íntegro proporciona uma mastigação com dinâmica e força adequadas e que o comprometimento do nervo trigêmio altera a sensibilidade e a ação dos músculos da face ${ }^{(21,24)}$.

Com relação à consistência alimentar oferecida, os resultados mostraram que o biscoito waffer e o pão francês (ambos sólidos, porém com consistências diferentes) tornaram difícil a mastigação, uma vez que o bolo alimentar ficou concentrado no vestíbulo oral, o que poderia levar à aspiração após a deglutição.

O pão de queijo, por sua vez, foi a consistência alimentar que exigiu mais força do masseter; porém, devido à sua consistência e textura, produziu um bolo alimentar mais coeso e não foram observados resíduos no vestíbulo oral e lateral após a mastigação.

Os mecanismos compensatórios observados durante a deglutição foram realizados para suprir a ineficiência da iner- vação da cavidade oral, laringe e faringe. Os pares de nervos cranianos V, VII, IX, X, XI e XII são essenciais e permitem que a mastigação e a deglutição sejam realizadas adequadamente $^{(7,27-30)}$.

O comprometimento do X par (vago) pode afetar a aferência das mucosas do véu palatino, laringofaringe, epiglote e da parte posterior da língua. Estas alterações podem contribuir para a aspiração devido à redução de sensibilidade geral da laringe e da valécula ${ }^{(29-30)}$. Este fenômeno, entretanto, não foi observado em nenhum paciente da amostra.

\section{CONCLUSÃO}

A análise dos resultados mostrou que as alterações morfofuncionais presentes nos pacientes com Sequência de Möbius contribuíram para a alteração da dinâmica da mastigação e deglutição. A mobilidade reduzida de língua contribuiu para a mastigação unilateral e a falta de contração do masséter reduziu a velocidade de mastigação.

Entre os sólidos que foram ofertados, o pão de queijo mostrou-se como o de melhor consistência, uma vez que produziu um bolo mais coeso e menos espalhado na cavidade oral. Tendo em vista este achado, é possível preparar refeições ou escolher alimentos que exijam menos esforços durante a mastigação e facilitem a deglutição.

Diante da dificuldade de ejeção oral, toda a amostra utilizou as manobras compensatórias durante a mastigação e deglutição. Mesmo com a ausência dos sinais clínicos sugestivos de penetração/aspiração, é de extrema importância que estes pacientes sejam submetidos à terapia fonoaudiológica, a fim de adequar os órgãos fonoarticulatórios na tentativa de proporcionar uma refeição mais prazerosa e com menos risco.

\section{AGRADECIMENTOS}

Ao Conselho Nacional de Desenvolvimento Científico e Tecnológico $(\mathrm{CNPq})$, pelo apoio concedido para realização desta pesquisa.

\begin{abstract}
Purpose: To describe morphofunctional alterations in the functions of chewing and deglutition, to recognize the most accepted food consistency, and to evaluate the presence of compensatory maneuvers during deglutition, in children and adolescents with Möbius syndrome. Methods: The subjects were eight children (three female and five male), with ages ranging from five to 15 years, diagnosed with Möbius syndrome. The children were evaluated during a feeding situation, where they were offered food with different consistencies: liquid, pasty and solid. Data were registered in an evaluation protocol adapted by Guedes, Shintani and Cabello (2003). Results: Results showed a significant relation between tongue mobility condition and type of mastication. The variables speed of mastication and contraction of the masseter muscle were also dependents ( $\mathrm{p}=0018)$, since there was no change in the speed of mastication when the contraction of the masseter was present. The cheese bread was the best solid consistency food for evaluation, because it produced a more cohesive and less widespread mass in the oral cavity. Conclusions: The morphofunctional alterations found in patients with Möbius syndrome contributed to change the dynamics of chewing and swallowing. All subjects in the sample used compensatory maneuvers. Therefore, even in the absence of clinical signs that suggest penetration/aspiration, patients with such diagnosis should be referred to speech therapy, in order to provide adequate conditions for a more pleasant meal.
\end{abstract}

Keywords: Möbius syndrome; Stomatognatic system; Deglutition; Mastication; Deglutition disorder 


\section{REFERÊNCIAS}

1. Guedes ZCF, Shintani MRS, Cabello S. Avaliação Estrutural e Funcional Fonoaudiológica de Órgãos Fonoarticulatórios em Crianças Portadoras de Paralisia Facial Congênita. [Protocolo utilizado no Ambulatório de Paralisia Facial da Universidade Federal de São Paulo - UNIFESP].

2. van der Bilt A, Engelen L, Pereira LJ, van der Glas HW, Abbink JH. Oral physiology and mastication. Physiol Behav. 2006;89(1):22-7. Review.

3. Woda A, Foster K, Mishellany A, Peyron MA. Adaptation of healthy mastication to factors pertaining to the individual or to the food. Physiol Behav. 2006;89(1):28-35.

4. Soboleva U, Laurina L, Slaidina A. The masticatory system - an overview. Stomatologija. 2005;7(3):77-80.

5. Guyton AC, Hall JE. Propulsão e mistura dos alimentos no trato alimentar. In: Guyton AC. Tratado de fisiologia médica. Rio de Janeiro: Elsevier; 2003. p. 781-5.

6. Ertekin C, Aydogdu I. Neurophysiology of swallowing. Clin Neurophysiol. 2003;114(12):2226-44.

7. Furlow B. Barium swallow. Radiol Technol. 2004;76(1):49-58; quiz 59-61.

8. Furkim AM, Santini CS. Deglutição - Normalidade. In: Furkim AM, Santini CSS. Disfagias orofaríngeas. 2a ed. São Paulo: Pró-Fono; 2004. p. 3-18.

9. Douglas CR. Tratado de fisiologia aplicada às ciências da saúde. São Paulo: Robe Editorial; 1994. cap.49. p. 857-81.

10. Yamada EK, Siqueira KO, Xerez D, Koch HA, Costa MMB. A influência das fases oral e faríngea na dinâmica da deglutição. Arq Gastroenterol. 2004;41(1):18-23.

11. Shaker R, Dodds WJ, Dantas RO, Hogan WJ, Arndorfer RC. Coordination of deglutitive glottic closure with oropharyngeal swallowing. Gastroenterology. 1990;98(6):1478-84.

12. Dantas R. A videofluroscopia no estudo das fases oral e faringeana da deglutição. Arq Gastroenterol. 1996;33(3):122-3.

13. Kidder TM. Esophago/pharyngo/laryngeal interrelationships: airway protection mechanisms. Dysphagia. 1995;10(4):228-31.

14. Imamura Y, Fujikawa Y, Komaki H, Nakagawa E, Sugai K, Sato N, Sasaki M. [A case of Möbius syndrome presenting with symptoms of severe infantile form of congenital muscular disorder]. No To Hattatsu. 2007;39(1):59-62. Japanese.

15. Strömland K, Sjögreen L, Miller M, Gillberg C, Wentz E, Johansson M, et al. Mobius sequence--a Swedish multidiscipline study. Eur J Paediatr Neurol. 2002;6(1):35-45.
16. Marques-Dias MJ, Gonzalez CH, Rosemberg S. Möbius sequence in children exposed in utero to misoprostol: neuropathological study of three cases. Birth Defects Res A Clin Mol Teratol. 2003;67(12):10027.

17. Gonzalez CH, Marques-Dias MJ, Kim CA, Sugayama SM, Da Paz JA, Huson SM, Holmes LB. Congenital abnormalities in Brazilian children associated with misoprostol misuse in first trimester of pregnancy. Lancet. 1998;351(9116):1624-7. Comment in: Lancet. 1998;352(9124):323. Lancet. 1999;353(9155):843-4.

18. Domingos AC, Lopes SL, Almeida SM, Boscolo FN, Whaites EJ. Poland-Moebius syndrome: a case with oral anomalies. Oral Dis. 2004;10(6):404-7.

19. Sensat ML. Mobius syndrome: a dental hygiene case study and review of the literature. Int J Dent Hyg. 2003;1(1):62-7.

20. Ha CY, Messieha ZS. Management of a patient with Möbius syndrome: a case report. Spec Care Dentist. 2003;23(3):111-6. Review.

21. Briegel W. Neuropsychiatric findings of Möbius sequence -- a review. Clin Genet. 2006;70(2):91-7.

22. Guedes ZCF. Atendimento fonoaudiológico das paralisias faciais no adulto e na criança. In: Lagrota MGM,César CPHAR. A fonoaudiologia nas instituições. São Paulo: Lovise; 1997. p. 163-7.

23. Allen BM, Wert MA, Tatum SA. Congenital unilateral multiple cranial neuropathy: an etiology shared with Mobius syndrome? Int J Pediatr Otorhinolaryngol. 2006;70(5):931-4.

24. De Serpa Pinto MV, De Magalhães MH, Nunes FD. Moebius syndrome with oral involvement. Int J Paediatr Dent. 2002;12(6):446-9.

25. Amaya LG, Walker J, Taylor D. Möebius syndrome: a study and report of 18 cases. Binocul Vis Q. 1990;5(3):119-32.

26. Sjögreen L, Andersson-Norinder J, Jacobsson C. Development of speech, feeding, eating, and facial expression in Möbius sequence. Int J Pediatr Otorhinolaryngol. 2001;60(3):197-204.

27. Sarnat HB. Watershed infarcts in the fetal and neonatal brainstem. An aetiology of central hypoventilation, dysphagia, Möbius syndrome and micrognathia. Eur J Paediatr Neurol. 2004;8(2):71-87.

28. Reed H, Grant W. Mobius's syndrome. Br J Ophthalmol. 1957;41(12):731-40.

29. Kuehn DP, Moon JB. Velopharyngeal closure force and levator veli palatini activation levels in varying phonetic contexts. J Speech Lang Hear Res. 1998;41(1):51-62.

30. Kuehn DP, Moon JB. Induced fatigue effects on velopharyngeal closure force. J Speech Lang Hear Res. 2000;43(2):486-500. 